\title{
Thickness dependence of space-charge-limited current in spatially disordered organic semiconductors
}

\author{
Muhammad Zubair, Member, IEEE, Yee Sin Ang, and Lay Kee Ang, Senior Member, IEEE
}

\begin{abstract}
Charge transport properties in organic semiconductors are determined by two kinds of microscopic disorders, namely energetic disorder related to the distribution of localized states and the spatial disorder related to the morphological features of the material. From a semi-classical picture, the charge transport properties are crucially determined by both the carrier mobility and the electrostatic field distribution in the material. Although the effect of disorders on carrier mobility has been widely studied, how electrostatic field distribution is distorted by the presence of disorders and its effect on charge transport remain unanswered. In this paper, we present a modified space-charge-limited current (SCLC) model for spatially disordered organic semiconductors based on the fractional-dimensional electrostatic framework. We show that the thickness dependence of SCLC is related to the spatial disorder in organic semiconductors. For trap-free transport, the SCLC exhibits a modified thickness scaling of $\mathrm{J} \propto \mathrm{L}^{-3 \alpha}$, where the fractional-dimension parameter $\alpha$ accounts for the spatial disorder in organic semiconductors. The trap-limited and field-dependent mobility are also shown to obey an $\alpha$-dependent thickness scaling. The modified SCLC model shows a good agreement with several experiments on spatially disordered organic semiconductors. By applying this model to the experimental data, the standard charge transport parameters can be deduced with better accuracy than by using existing models.
\end{abstract}

\section{INTRODUCTION}

The mobility of charge carrier is a key parameter for the performance of optoelectronic devices [2], especially for devices using organic semiconductors and polymers. The mobility in organic semiconductors strongly depends on the nature, structure, purity of the materials and device operating conditions. The charge transport in organic compounds occurs across various levels, ranging from within molecules, between molecules as well as between crystalline grains and amorphous and crystalline regions. The transport properties

Muhammad Zubair is with the Department of Electrical Engineering at Information Technology University of the Punjab, Ferozpur Road, 54000 Lahore, Pakistan. He was affiliated until recently with the SUTD-MIT International Design Center, Singapore University of Technology and Design, 487372 Singapore (e-mail: muhammad.zubair@itu.edu.pk).

Yee Sin Ang, and Lay Kee Ang are affiliated with the SUTDMIT International Design Center, Singapore University of Technology and Design, 487372 Singapore (e-mails: yee_sin@sutd.edu.sg, ricky_ang@sutd.edu.sg).

The authors are thankful to P. W. M. Blom for providing the experimental data of Ref. [1] and helpful discussion. This work is sponsored by USA AFOSR AOARD (FA2386-14-1-4020), Singapore Temasek Laboratories (IGDS S16 0205 1) and A*STAR IRG (A1783c0011). are determined by two kinds of microscopic disorders, namely the energetic disorder characterized by a broad distribution of localized states and the spatial disorder related to the morphological features of the material [3]. The space-chargelimited current (SCLC) is an important classical transport phenomenon in organic semiconductors where the quantum effects can be ignored at microscale and above [1]-[17].

The mobility of a given organic material sandwiched between two planar electrodes with an applied voltage $\left(V_{0}\right)$, is commonly measured indirectly by fitting the measured current density-voltage (J-V) characteristics at high voltages to some SCLC models [4]. It is assumed that there is no barrier (ohmic contact) at the interface when the charges are injected from the electrode into the solid. The simplest SCLC model for a trap-free solid is known as the one-dimensional (1D) classical Mott-Gurney (MG) law [5], given by

$$
J=\frac{9}{8} \epsilon \mu \frac{V_{0}^{2}}{L^{3}},
$$

where $\epsilon=\epsilon_{0} \epsilon_{r}$ is permittivity, and $L$ is the thickness of the solid embedded between the metal electrodes. Once the values of $J, V_{0}, L$ and $\epsilon$ are determined, the mobility $\mu$ can be calculated by fitting the $\mathrm{J}-\mathrm{V}$ characteristics at high $V_{0}$ region to the 1D MG law. Note the assumptions in using the MG law include constant mobility (independent of the applied electric field and charge density) and the solid is trap-free.

For a trap-filled solid with exponentially energy-distributed traps, the corresponding SCLC model is known as the traplimited (TL) SCLC model [6]:

$$
J=N_{c} \mu e^{1-l}\left[\frac{\epsilon l}{N_{t}(l+1)}\right]^{l}\left(\frac{2 l+1}{l+1}\right)^{l+1} \frac{V_{0}^{l+1}}{L^{2 l+1}},
$$

where $N_{c}$ is the effective density of states corresponding to the energy at the bottom of the conduction band, $N_{t}$ is the total trapped electron density, and $l=T_{t} / T \geq 1$ with $T_{t}$ being a parameter controlling the trap distribution.

For shallow traps or energetic disorder, the mobility varies with the electric field $E$ that Murgatroyd's [7] model may be used to describe a field-dependent mobility in the form of

$$
\mu=\mu_{0} \exp (\gamma \sqrt{E}),
$$

where $\mu_{0}$ is representing the mobility at zero field, and $\gamma$ is a material-specific parameter that describes the strength of the field-dependence. The field-dependent mobility can 
also include other effects, such as carrier-density dependence (Gaussian disorder model (GDM) [8]) and deep traps, which can only be resolved by having a more comprehensive model to fit with experiments over a wide range of parameters [9]. By using Eq. [3), we get [7]

$$
J=\frac{9}{8} \epsilon \mu_{0} \frac{V_{0}^{2}}{L^{3}} \exp (0.89 \gamma \sqrt{E}),
$$

where $0.89 \gamma$ is used as a fitting parameter.

Other than field-dependent mobility, charge-carrier-density dependent mobility models have also been studied including a power-law dependence for energetically disordered semiconductors by Tanase et. al. [10], Blom et. al. [1], and others for disordered polymers [11]-[13].

The mobility is often extracted from $\mathrm{J}-\mathrm{V}$ measurements by fitting the experimental data with the theoretical models of SCLC with different mobility terms. The mobility of a given sample is determined solely based on the goodness of the fit. Such empirical methodology may not always produce accurate physical picture. For example, an inconsistency of field-dependent mobility model with the experimental data has been raised in Ref. [1]. The conventional model of Eq. (4) could not fit the experimental data due to weaker thickness dependence of measured SCLC, and hence the carrier-density dependent mobility model was chosen to extract the mobility of PPV based diode device. However, assuming Gaussian density of states (DOS), it is known that in low carrierdensity regime the mobility should be carrier-density independent [14]. Another recent experiment [3] has also reported that the charge transport in amorphous semiconductors is not charge density dependent but instead follows a field-dependent mobility model. In such scenarios, a new model of spacecharge-limited transport is required to capture the correct thickness scaling of measured SCLC.

The transport sites in organic semiconductors are distributed both in space and energy. The combined effect of spatial and energetic disorder on charge transport has already been studied in the previous works (see [18] for a comprehensive review). However, the SCLC is a transport phenomenon closely related with both the material properties (i.e., mobility) and the electrostatic field distribution inside these materials, and thus far, the non-uniform distribution of electrostatic field due to spatial complexity of the material and its implications on the macroscopic SCLC have not been fully addressed. The complex, spatially disordered and often self-organized microstructure, in which ordered microcrystalline domains are embedded in an amorphous domain, can be considered as fractal features having important consequences for electrical properties of these materials (refer to Fig. 2 in [19] and Fig. 1 in [20]).

In this work, we present a modified SCLC model to account for the spatial disorder effect of a solid such as amorphous semiconducting polymer by treating the material as a fractal object. The key novelty of our proposed model is to utilize the fractional-dimension of space to effectively model the nonuniform distribution of electrostatic field inside these spatially disordered materials. Fig. (17) provides a schematic description of the concept that the spatially disordered organic semicon- ductor in real integer-dimensional space can be considered effectively as spatially ordered organic semiconductor embedded in the corresponding fractional-dimensional space using mathematical framework briefly introduced in the Appendix (also see [21], [22] and references therein). Such methods have been applied in other areas including quantum field theory [23], [24], general relativity [25], thermodynamics [26], mechanics [27], hydrodynamics [28], electrodynamics [29][38], and fractional charge transport [21], [22] to name a few.

The proposed approach has been generalized to cover three types of SCLC models: trap-free model (or MG law), traplimited (TL) model and the field dependent mobility models. We first analyze various experimental results to study the thickness (or $L$ ) dependence to show that the traditional $L$ scalings from the traditional models are not valid for spatially disordered semiconducting materials. By using our proposed models, we are able to reproduce the experimental currentvoltage measurements in [1] without using the carrier-density dependent model, and thus solving the issues raised by recent paper [3].

\section{DeRivation OF SCLC SCALING LAWS FOR SPATIALLY DISORDERED ORGANIC SEMICONDUCTORS}

\section{A. Trap-free model}

Here, we derive the modified MG law for spatially disordered organic semiconductors with the assumption that the effect of non-uniform electrostatic field distribution inside spatially disordered material can be studied effectively by replacing the governing equations of classical SCLC model with the fractional-dimensional counterparts, by using the formulation described in Appendix (for more details see [21], [22] and references therein), where the fractional-dimension is related to the amount of spatial disorder. Provided that the thermal carriers are negligible in comparison to injected carrier, and assuming that the size of the electrode is much greater than the spacing $L$, thus the derivation is conducted only in $x$ direction perpendicular to surface of the electrode. The following equations are solved in the $\alpha$-dimensional space [39] with $0<\alpha \leq 1$ :

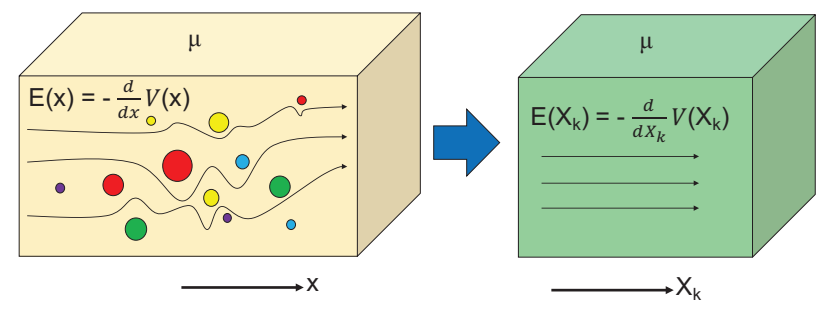

Fig. 1. Schematic description of the concept that the spatially disordered organic semiconductor in real space [shown on left] can be considered effectively as spatially ordered organic semiconductor embedded in the corresponding fractional-dimensional space [shown on right] using fractional-dimensional space framework as description of complexity described in Appendix. $\boldsymbol{X}_{\boldsymbol{k}}$ corresponds to the spatial coordinates of an $\boldsymbol{\alpha}$-dimensional space. 


$$
\begin{array}{r}
J=-\rho \nu, \\
\nabla_{\alpha} \cdot E=\frac{1}{c(\alpha, x)} \frac{d E}{d x}=\frac{\rho}{\epsilon}, \\
E=-\nabla_{\alpha} V=-\frac{1}{c(\alpha, x)} \frac{d V}{d x},
\end{array}
$$

where $c(\alpha, x)=\frac{\pi^{\alpha / 2}}{\Gamma(\alpha / 2)}|x|^{\alpha-1}[21], \rho$ is the carrier charge density, $\nu$ is the drift velocity, $E$ is the electric field, and $\epsilon$ is the dielectric permittivity of the material, and $V$ is the electric potential. Using $\nu=-\mu E$, Eq. (5) gives

$$
J=\rho \mu E \text {. }
$$

It should be emphasized that $\mu$ is an averaged quantity independent of space variables.

Now, inserting Eq. (6) into Eq. (8), we get

$$
J=\epsilon \mu \frac{1}{c(\alpha, x)} E \frac{d E}{d x},
$$

which can be rewritten in the form of Bernoulli differential equation:

$$
\frac{d E}{d x}=\frac{c(\alpha, x)}{\epsilon \mu} J E^{-1} .
$$

Solving Eq. (10) with zero electric field condition (at SCL regime) at the injecting electrode, $E(0)=0$, we have

$$
E(x)=\sqrt{\frac{2 J x^{\alpha} \pi^{\alpha / 2}}{\alpha \epsilon \mu \Gamma(\alpha / 2)}} .
$$

By using Eq. (11) in solving Eq. (7), we obtain

$$
V(x)=\sqrt{\left[\frac{\pi^{\alpha / 2}}{\Gamma(\alpha / 2)}\right]^{3} \frac{J}{\epsilon \mu} x^{3 \alpha} .}
$$

which gives the modified MG law as a function of $\alpha$ :

$$
J=\frac{9 \alpha^{3}}{8}\left[\frac{\Gamma(\alpha / 2)}{\pi^{\alpha / 2}}\right]^{3} \epsilon \mu \frac{V_{0}^{2}}{L^{3 \alpha}} .
$$

For $\alpha=1$, Eq. (13) reduces to the classical MG Law [Eq. [1].

\section{B. Trap-limited (TL) model}

For a spatially disordered material with exponentially distributed traps in energy, the trap-limited TL-SCLC injection is derived here. We assume that the mobility is field-independent, and that the density of the trapping states per unit energy range $h(E)$ above the valence band is described by the distribution

$$
h(E)=\left(N_{t} / k T_{c}\right) \exp \left(-E / k T_{c}\right),
$$

where $E$ is the energy measured upward from the top of the valence band, $N_{t}$ is the total trap density, and $T_{c}$ is a characteristic constant of the distribution. Following the Mark and Helfrich $(\mathrm{MH})$ approach [6], we obtain

$$
\rho(x)=\left(\frac{J}{N_{c} \mu}\right)^{1 / l} e_{0}^{l-1 / l} N_{t} E^{-1 / l},
$$

where $l=T_{c} / T$ and $N_{c}$ is effective density of states. In this case the relation between free $n$ and trapped carrier density $n_{t}$ is given by

$$
\frac{n}{N}=\left(\frac{n_{t}}{N_{t}}\right)^{l}
$$

where, $N$ is total density of transport sites. By solving Eq. (6), the governing equation is

$$
\begin{array}{r}
\frac{d E(x)}{d x}=F \frac{x^{\alpha-1}}{[E(x)]^{1 / l}}, \\
F=\left(\frac{J}{N_{c} \mu}\right)^{1 / l} e_{0}^{l-1 / l} N_{t} / \epsilon \frac{\pi^{\alpha / 2}}{\Gamma(\alpha / 2)} .
\end{array}
$$

Integrating Eq. (17) on both sides gives

$$
E(x)=\left(\frac{l+1}{l} \frac{F}{\alpha}\right)^{\frac{l}{l+1}} x^{\frac{\alpha l}{l+1}},
$$

and

$$
V(x)=\frac{\pi^{\alpha / 2}}{\Gamma(\alpha / 2)} \int E(x) x^{\alpha-1} .
$$

The analytical evaluation of above integral leads to

$$
\begin{aligned}
J= & N_{c} \mu e^{1-l}\left[\frac{\Gamma(\alpha / 2)}{\pi^{\alpha / 2}}\right]^{2 l+1}\left[\frac{\epsilon \alpha l}{N_{t}(l+1)}\right]^{l} \\
& \times\left(\frac{2 \alpha l+\alpha}{l+1}\right)^{l+1} \frac{V_{0}^{l+1}}{L^{2 \alpha l+\alpha}},
\end{aligned}
$$

which reduces to Eq. (2) at $\alpha=1$.

It should be noted that for a Gaussian distribution of traps, a similar equation for the trap-limited current is derived, except that $l$ is then related to the depth and width of the trap distribution [40], [41]. In the case of Gaussian trap DOS centered at a distance $E_{t c}-E_{a}$ below the conduction-band edge ,the nondegenerate approximation gives [42]

$$
\frac{n}{N}=\left(\frac{n_{t}}{N_{t} \exp \left(\left(E_{t c}-E_{a}\right) / k T_{c}\right)}\right)^{l},
$$

where, $E_{a}=\frac{\sigma^{2}}{2 k T}, \sigma$ is the variance of Gaussian DOS. Finally, following the $\mathrm{MH}$ formalism, a current-voltage characteristic is obtained for Gaussian trap DOS, which is of the form

$$
\begin{aligned}
J= & N_{c} \mu e^{1-l} \\
& \times\left[\frac{\Gamma(\alpha / 2)}{\pi^{\alpha / 2}}\right]^{2 l+1}\left[\frac{\epsilon \alpha l}{N_{t} \exp \left(\left(E_{t c}-E_{a}\right) / k T_{c}\right)(l+1)}\right]^{l} \\
& \times\left(\frac{2 \alpha l+\alpha}{l+1}\right)^{l+1} \frac{V_{0}^{l+1}}{L^{2 \alpha l+\alpha}},
\end{aligned}
$$

which reduces to Eq. (7) in [42] at $\alpha=1$.

\section{Field-dependent mobility model}

If we simply combine the field-dependent mobility Eq. (3), and Eq. (13), the modified SCL model of field-dependent mobility for spatially disordered semiconductors is

$$
J=\frac{9 \alpha^{3}}{8}\left[\frac{\Gamma(\alpha / 2)}{\pi^{\alpha / 2}}\right]^{3} \epsilon \mu_{0} \frac{V_{0}^{2}}{L^{3 \alpha}} \exp (0.89 \gamma \sqrt{E})
$$


where $\gamma$ is just a fitting parameter. In general, to include the field dependence of the mobility in SCLC model, coupled equations such as Eqs. (5.77) must be solved consistently [43]. It is however possible to derive an analytic solution if the field dependence of the drift mobility can be expressed in power law [44] given by

$$
\mu=\mu_{0}\left(\frac{E}{E_{0}}\right)^{n},
$$

with $\mu=\mu_{0}$ at $E=E_{0}$. By using this power law of mobility, we solve Eqs. (5.77) to obtain an analytical solution of

$$
\begin{aligned}
J= & \frac{\epsilon \mu_{0}}{E_{0}^{n}}\left[\frac{\Gamma(\alpha / 2)}{\pi^{\alpha / 2}}\right]^{n+3}\left[\frac{n \alpha-n+\alpha}{n+2}\right] \\
& \times\left[\frac{2 n \alpha+3 \alpha-n}{n+2}\right]^{n+2} \frac{V_{0}^{n+2}}{L^{2 n \alpha+3 \alpha-n}},
\end{aligned}
$$

which reduces to Eq. 13 at $n=0$.

\section{Results And Discussions}

By analyzing the thickness $(L)$ dependence of the classical SCLC models, we see the dependence of $L^{-3}$ (at fixed $V$ ), $L^{-2 l-1}$ (at fixed $V$ ) and $L^{-1}$ (at fixed $E$ ), respectively, from Eq. (1), Eq. (2), and Eq. (4). However, as predicted by corresponding modified SCLC models in Sec. II, the thickness dependence will be reduced by the fractional-dimension parameter $\alpha$, which accounts to the spatial disorder in the underlying solids. In other words, the thickness dependence of the modified SCLC models will provide a tool to characterize the spatial disorder in the porous organic semiconductor.

\section{A. Implications of modified SCLC model on mobility extraction}

Before proceeding with the analysis of thickness dependence in some reported experimental data, it would be of interest to see the effect of variation in thickness dependence due to spatial disorder in the semiconductors on the mobility values extracted form experimental J-V curves taken from [1]. We denote the extracted values of mobility by $\tilde{\mu}$, to distinguish them from actual mobility $\mu$ for this device. In Fig. (2), the extracted mobility $(\tilde{\mu})$ is plotted as a function of thickness dependence parameter $3 \alpha$. The fractional-dimension parameter $\alpha$ corresponds to the measure of spatial disorder in the semiconductor, with $\alpha=1$ corresponding to zero spatial disorder. It can be seen that $\tilde{\mu}$ is sensitively influenced by $\alpha$. Thus it is important to check the $L$ dependence rather than assuming $\alpha=1$ which may no longer be valid for complicated materials such as porous and amorphous organic materials.

\section{B. Consistency of $\alpha$ extracted from experimental data}

Most organic semiconductors have spatial as well as energetic disorder. The existing mobility models incorporate combined effect of energetic and spatial disorder using a range of mobility models including field- and density-dependent mobility. However, our model predicts that the spatial disorder can affect the thickness scaling of SCLC. Here, we explore the available experimental data of SCLC versus device

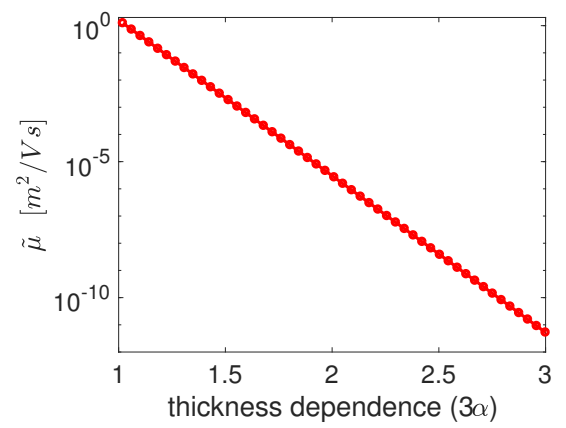

Fig. 2. The variation of the mobilities of PPV based devices as a function of varying thickness dependence $\left(L^{3 \alpha}\right)$. It is shown that a variation in thickness dependence leads to several orders of under- or over-estimation of mobility values. The $\mathrm{J}-\mathrm{V}$ data is taken from [1] for $\boldsymbol{L}=950 \mathrm{~nm}$

thickness for a range of disordered organic semiconductors. It is observed that the thickness scaling of SCLC varies as predicted by our model, to which not much attention was paid previously and it was assumed trivially that thickness scaling follows standard MG law which turns out to be not true for organic semiconductor in several example cases reported in the following. Fig. (3) shows the corresponding thickness $L$ dependence for various devices using different organic materials. The results shown in Fig. (3a-3d) are at fixed voltage regime (constant mobility) with varying thickness $L$. Based on the classical MG law, we will expect a scaling of $L^{-3}$. However, due to spatial disorder, the results show a weaker thickness dependence in the range $L^{-2}$ to $L^{-3}$, which corresponds to about $\alpha \approx 0.8$ to $\alpha \approx 1$.

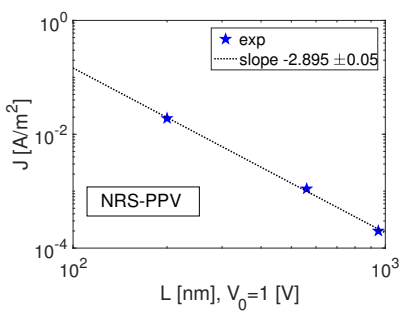

(a)

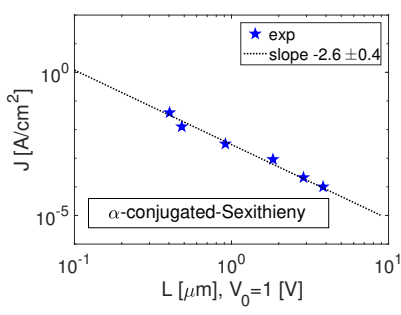

(c)

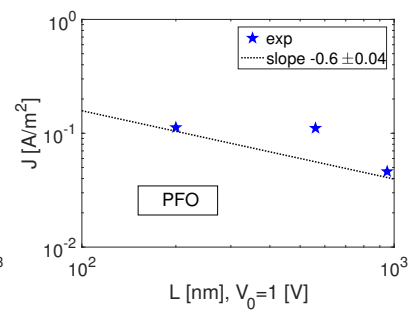

(b)

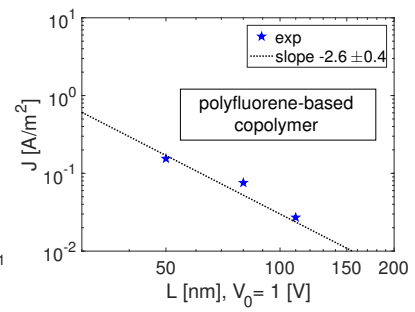

(d)
Fig. 3. The thickness dependency of the current density at fixed voltage for different polymers taken from current-voltage characteristics in the literature: (a) $N R S-P P V$ [1] (b) $P F O$ [45] (c) $\alpha$-conjugatedSexithienyl [46] (d) poly-fluorene-based [47].

For the results shown in Fig. 4a for a trap-filled organic material we have $l=T_{t} / T=1500 / 273=5.49$. Based on the classical TL-SCLC model (without any spatial disorder), the thickness dependence should be $L^{-11.98}$. However, the 
TABLE I

THICKNESS DEPENDENCE OF ORGANIC SEMICONDUCTOR DEVICES BASED ON DISORDERED SEMICONDUCTING ORGANIC POLYMERS AND ITS RELATION TO PARAMETER $\boldsymbol{\alpha}$ USED IN MODIFIED SCLC MODELS.

\begin{tabular}{lllll}
\hline \hline Polymer Type & $\begin{array}{l}\text { Average thickness } \\
\text { dependence } \\
\text { (extracted from } \\
\text { experiment) }\end{array}$ & $\begin{array}{l}\text { Thickness dependence } \\
\text { relation } \\
\text { (modified MG law) }\end{array}$ & $\begin{array}{l}\text { Calculated } \alpha \\
\text { (measure of spatial } \\
\text { disorder) }\end{array}$ & $\begin{array}{l}\text { Ref. } \\
\text { (experimental } \\
\text { data) }\end{array}$ \\
\hline \hline NRS-PPV & 2.895 (Fig. (3a)) & $3 \alpha$ (Eq. (19)) & 0.965 & {$[1]$} \\
PFO & 2.9 (Fig. (3b)) & $3 \alpha$ & 0.967 & {$[45]$} \\
$\alpha$-conjugated-Sexithienyl & 2.6 (Fig. (3c)) & $3 \alpha$ & 0.86 & {$[46]$} \\
poly-fluorene-based & 2.5 (Fig. (3d)) & $3 \alpha$ & 0.83 & {$[47]$} \\
$O C_{1} C_{10}-P P V$ & 11 (Fig. (4a)) & $2 \alpha l+\alpha$ (Eq. (27)) & 0.918 & {$[48]$} \\
NPB & 0.52 (Fig. (4b)) & $3 \alpha$-2 (Eq. (30)) & 0.84 & {$[49]$} \\
\hline \hline
\end{tabular}

experimental fitting shows again a weaker dependence, which corresponds to $\alpha=0.918$ based on Eq. (21), instead of $\alpha=$ 1. For results shown in Fig. $4 \mathrm{bb}$, the $L$ scaling is calculated from measurements at fixed electric field. As mentioned earlier for field-dependent mobility, the thickness dependence should be $L^{-1}$ at a fixed field for negligible spatial disorder at $\alpha=1$. However, we observe a weaker dependence, which corresponds to $\alpha=0.86$ based on Eq. 247). Table I summarizes the results of Fig. (34) for various disordered organic semiconductor based devices and its relation to the $\alpha$ parameter used in our models to fit with the experimental results. Our analysis suggests that the traditional $L$ scaling formulated in the classical SCLC models may not be suitable for organic semiconductors, and it will provide an inaccurate estimation of the mobility if such models are used. Note that the uncertainty in the measurement of $L$, which is about $5 \mathrm{~nm}$ from normal experimental setup, is not able to explain the variation from the expected $\alpha=1$ assuming the classical models are correct. (a)

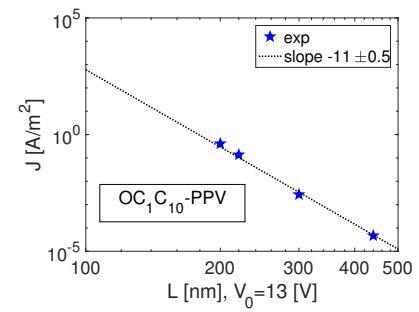

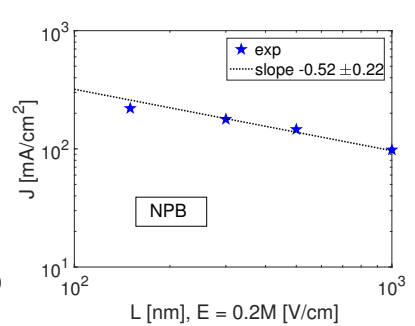

(b)
Fig. 4. The thickness dependency of (a) trap-limited current density at fixed voltage for polymer $O_{1} C_{10}-P P V$ taken from current-voltage characteristics in the literature [48] (b) current density at fixed electric field for polymer $N \boldsymbol{P B}$ taken from current-field characteristics in the literature [49].

In Fig. (3), we have extracted the thickness scaling of SCLC at low voltages to avoid the field-dependent SCLC regime. However, one must be careful while extracting the slopes at higher applied voltages. As the SCLC is fielddependent at high applied voltage, the extraction of $\alpha$ should be performed at fixed electric field strength, $E$, rather than at fixed voltage, $V$. To demonstrate this, we analyze the SCLC versus voltage data of polymer NPB based devices reported in Ref. [49], and plot the current density against device thickness at different voltages in Fig. 5a. The varying slope of $J$ versus $L$ shows that the thickness dependence of SCLC varies at different applied voltages due to $\exp (\gamma \sqrt{V / L})$ factor in field-dependent mobility model of Eq. 24). Fig. (5b) shows the extracted thickness dependence at different voltages which immediately reveals that the value of the extracted $\alpha$ is inconsistent at different voltage. At high-voltage regime where field-dependence becomes non-negligible, the extracted thickness dependence even becomes stronger than $L^{-3}$ which leads to an unphysical value of $\alpha>1$. This clearly reveals the fallacy of extracting $\alpha$ from the J-L curve at fixed voltage. Instead, the $\alpha$ should be extracted at fixed electric field strength as indicated by Eq. 24), i.e., $J \propto L^{3 \alpha-2}$ at fixed $E$. Fig. (5c) and (5d) shows the J-L characteristics and the extracted $\alpha$ at different $E$, respectively. In this case, a singular value of $\alpha \approx 0.84$ is extracted for all applied electric field strengths. More importantly, this value of $\alpha$ is consistent with that extracted from the low-voltage regime of Fig. (5b).

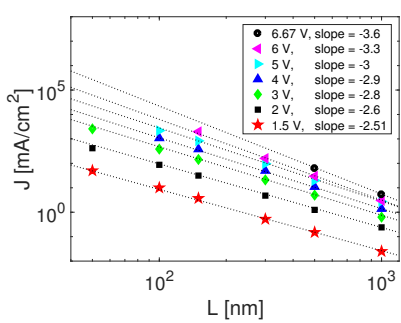

(a)

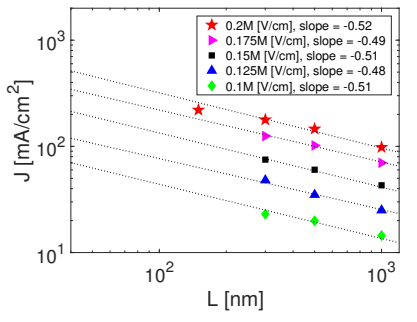

(c)

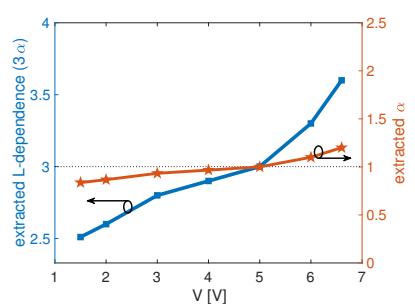

(b)

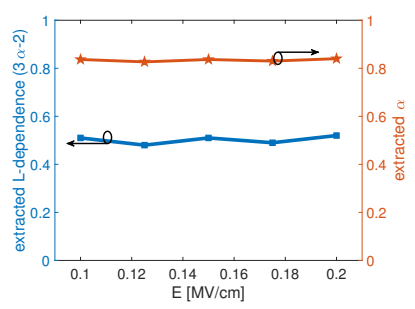

(d)
Fig. 5. Experimental data for polymer $\boldsymbol{N P B}$ based devices taken from [49]. (a) The current density versus device thickness for varying voltages (applied voltage $\boldsymbol{V}$ and slope is shown in legend). (b) The extracted thickness dependence and the parameter $\boldsymbol{\alpha}$ at different voltages. (c) The current density versus device thickness for varying electric field (applied electric field $\boldsymbol{E}$ and slope is shown in legend). (d) The extracted thickness dependence and the fractional dimension parameter $\alpha$ at different applied electric fields. 
In Fig. 6a), the room-temperature current density versus voltage characteristics data from Ref. [45] is shown for PFO diodes of varying thickness together with various numerical models calculations. It should be noted that the classical model of Eq. (4) requires different values of $\gamma$ to be used in order to fit with the experimental data despite the fact that the devices are composed of the same type of polymer. To address this inconsistency, we fitted the experimental data using our modified SCLC model with $\alpha=0.967$ [extracted from Fig. [3b]]. Remarkably, our modified model is able to fit the experimental $\mathrm{J}-\mathrm{V}$ curves of all devices using a singular consistent value of $\gamma=1.2 \times 10^{-4}(\mathrm{~m} / \mathrm{V})^{1 / 2}$. Similarly, in Fig. (6b) the room-temperature current density versus voltage data from Ref. [48] for $O C_{1} C_{10}-P P V$ diodes with varying thicknesses is shown. The classical model in Eq. (2) fails to reproduce the experimental results with $N_{t}$ fixed for all L. In contrast, by using our modified trap-limited SLC model with $\alpha=0.918$ extracted from Fig. (4a), a much better agreement with experimental results is obtained at a fixed $N_{t}$. These results show that the modified MG law can sufficiently describe the thickness dependence of SCLC for given range of applied voltages.

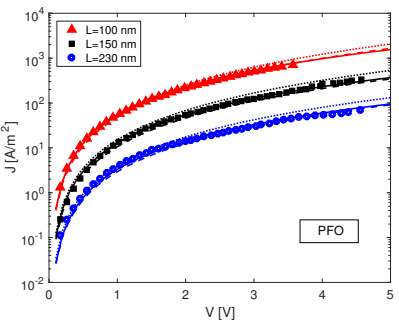

(a)

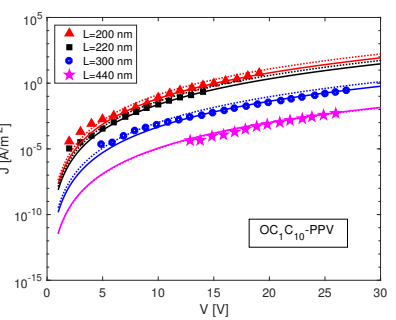

(b)
Fig. 6. (a) Room-temperature current density vs voltage characteristics data from [45] for $\boldsymbol{P F O}$ diodes with thicknesses of 100 (red), 150 (black), and 250 (blue) nm, respectively. Experiment (circles), Eq. 4 (dotted lines), Eq.24 (dashed lines), Eq. 26 (solid lines). The parameters used are $\gamma=1.2 \times 10^{-4}(\mathrm{~m} / \mathrm{V})^{1 / 2}, \mu_{0}=1.3 \times 10^{-9} \mathrm{~m}^{2} / V \mathrm{~s}$, $\alpha=0.967, E_{0}=0.1 / L^{\alpha}, n=0.088$. (b) Room-temperature current density vs voltage characteristics data from [48] for $O C_{1} C_{10}-$ $\boldsymbol{P P V}$ diodes with thicknesses of 200 (red), 220 (black), 300 (blue), and 440 (green) $\mathrm{nm}$ respectively. Experiment (circles), Eq. 2 (dotted lines), Eq. 21 (solid lines). The parameters used are $\alpha=\mathbf{0 . 9 1 8}$, $N_{t}=8.5 \times 10^{23} \mathrm{~m}^{-3}, T_{t}=1500 \mathrm{~K}$ and zero-field mobility of $5 \times 10^{-11} m^{2} / V s$.

\section{Fitting experimental current-voltage characteristics and mobility extraction using modified SCLC model}

In Fig. (7a) the experimental J-V characteristics [1] (circles) of the NRS-PPV based devices are shown together with various numerical models calculations: (i) (dotted lines) classical MG law based field-dependent mobility model of Eq. (4), (ii) (dashed lines) modified MG law based field-dependent mobility model of Eq. (24), and (iii) (solid lines) modified MG law based field-dependent mobility model of Eq. 26. From the figure, it is clear that the classical model (dotted lines) does not have a good agreement with the experimental results. As shown in Fig. (3a), NRS-PPV based devices show a thickness dependence of $L^{-2.895}$ which corresponds to $\alpha=0.965$. Using this $\alpha=0.965$, the two modified
SCL models including field dependent mobility (dashed and solid lines) are able to provide better agreements without the needs to use carrier-dependent mobility assumption that have been debated in recent years [18]. It is important to note that one of the direct consequence of modified MG law in Eq. (24) is that the mobility can be considered to have a thickness dependence along with field-dependence given by $\mu=\mu_{0} \exp (0.89 \gamma \sqrt{E}) L^{3-3 \alpha}$. In Fig. 7 b) we show the field and thickness dependent mobility values for the same NRSPPV based devices [1] using this model with the parameters shown in figure caption.

Finally, we analyzed the thickness dependence of experimentally measured SCLC in hole-only devices based on diketopyrrolopyrole-based polymer (PDPPDTSE) [50]. The thickness dependence of current density at fixed voltage for PDPPDTSE based devices taken from experimental currentvoltage data is shown in Fig. 8a). The thickness scaling of SCLC from standard $L^{-3}$ to $L^{-1.07}$. The observed thickness dependence corresponds to spatial disorder parameter $\alpha=$ 0.3567 . In order to validate our model we also compared the reported mobility values for varying thickness of devices with the mobility scaling predicted by our model. It is shown in Fig. $(8 \mathrm{~b})$ that the thickness scaling of measured mobility is in good agreement with the one predicted by our model $\left(L^{3-3 \alpha}\right)$.

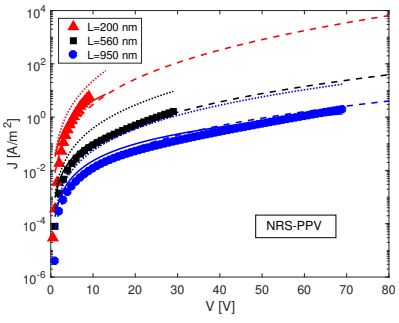

(a)

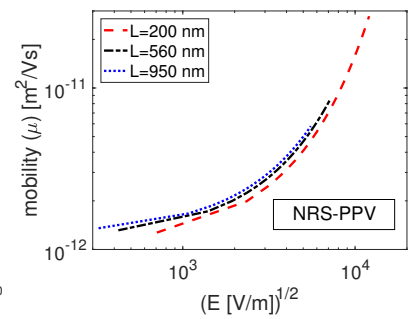

(b)
Fig. 7. (a) Room-temperature current density vs voltage characteristics data from [1] for NRS-PPV hole-only diodes with thicknesses of 200 (red), 560 (black), and 950 (blue) nm, respectively. Experiment (circles), Eq. 4 (dotted lines), Eq. 24 (dashed lines), Eq. 26 (solid lines). The parameters used are $\gamma=4 \times \mathbf{1 0}^{-4}(m / V)^{I / 2}, \mu_{0}=5 \times$ $10^{-12} m^{2} / V s, \alpha=0.965, E_{0}=0.1 / L^{\alpha}, n=0.10$. (b) The calculated mobility values for devices with varying thickness using $\mu=\mu_{0} \exp (0.89 \gamma \sqrt{E}) L^{3-3 \alpha}$.

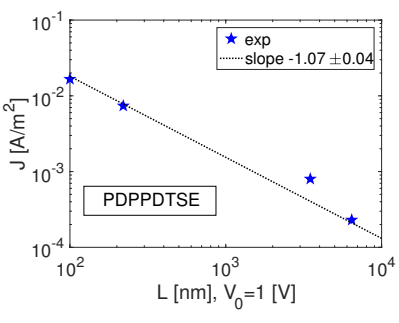

(a)

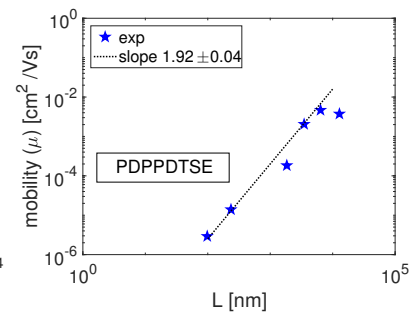

(b)
Fig. 8. (a) The thickness dependence of current density at fixed voltage for diketopyrrolopyrole-based polymer (PDPPDTSE) taken from experimental current-voltage data in the literature [50]. The observed thickness dependence corresponds to spatial disorder parameter $\boldsymbol{\alpha}=$ 0.3567. (b) The thickness dependence of measured mobility agrees with the one predicted by our model $\left(L^{3-3 \alpha}=L^{1.929}\right)$. 


\section{SUMmaRY}

In summary, we have presented a modified thickness scaling in SCLC model to account for the spatial disorder in organic semiconductors by introducing a parameter $\alpha$ to imagine the solid as a fractal object sandwiched between two electrodes. The model has included different effects such as trap-free, trap-limited and field-dependent mobility. To provide an easy access to the main results of this work, we have summarized the modified SCLC equations in Table II. An analysis of multiple experimental results from literature reveals that the classical SCLC models might lead to incorrect extraction of mobilities due to weak thickness dependence arising from spatial disorder. For such materials, our proposed model here would be a better choice to extract the mobility for spatially disordered organic materials as we have shown that the traditional thickness scaling is not valid anymore. By applying our model with field-dependent mobility, we are able to reproduce the experimental results of SCLC transport in PPV derivative based device without using the carrier-density dependent mobility [1], agreeable with a recent report for amorphous polymers [3].

Note that the thickness dependence had been reported in others works. For example, Brutting et. al (see Fig. 2a in Ref. [51]) reported a weaker thickness dependence for Alq light-emitting devices than the expected $L^{-1}$ at fixed electric field. John et. al (see Fig. (5-6) in Ref. [52]) reported a varying thickness dependence $\left(L^{-2.7 \pm 0.46}\right.$ to $\left.L^{-3.14 \pm 0.7}\right)$ for plasma polymerized pyrrole thin films. Boni et. al. (see Fig. 12 in Ref. [53]) also reported a possible weaker thickness dependence for PZT ferroelectric based devices. Macdonald et. al (see Fig. 1b in Ref. [54]) also reported a weaker thickness dependence due to non-planar electrodes in conducting the experiment using tip atomic force microscopy (cAFM). This is a geometrical effect producing weaker thickness dependence of organic semiconductor devices [55] and is different from the physics studied here. It should be emphasized that our proposed models are based on a planar-diode geometry, thus such non-planar geometrical effects are not included. The extension of our models into non-planar geometries will be pursued in future works.

Moreover, in this work we obtain the parameter $\alpha$ from the length scaling of SCLC in the experimental results, however a complete microscopic model can be created in further extensions to determine $\alpha$ directly from the knowledge of disorder either spatial or energetic or both.

\section{APPENDIX}

\section{FRACTIONAL-DIMENSIONAL SPACE FRAMEWORK AS DESCRIPTION OF COMPLEXITY}

There is an increasing interest in the fractional modeling of complexity in physical systems [56], [57]. In recent years, the concept of fractional-dimensional space has been used as an effective physical description of restraint conditions in complex physical systems [24], [29], [39]. The approaches to describe the fractional dimensions include fractal geometry [58], fractional calculus [59], [60], and the integration over fractional-dimensional space [23], [61]. The axiomatic basis of spaces with fractional dimension with Euclidean metric were introduced by Stillinger [23]. The fractionaldimensional generalization of first order Laplace operators was then reported by Zubair et. al. [29] as approximations of the square of the second-order Laplace operator introduced in [23], [24]. Recently, a fractal metric based approach is considered by Tarasov [39] which provides a complete generalization of first and second order Laplace operators. In this work, we have utilized Tarasov's approach to vector calaculus in fractionaldimensional spaces, which is summarized in the following.

In fractional-dimensional space $\left(F^{\alpha} \subseteq E^{n}\right)$ framework [39], it is convenient to work with physically dimensionless space variables $x / R_{0} \rightarrow x, y / R_{0} \rightarrow y, z / R_{0} \rightarrow z$, $\mathbf{r} / R_{0} \rightarrow \mathbf{r}$, where $R_{0}$ is a characteristic size of considered model. This provides a dimensionless integration and differentiation in $\alpha$-dimensional space which leads to correct physical dimensions of quantities.

We define a differential operator in the form of

$$
\partial_{\alpha_{k}, x_{k}}=\frac{\partial}{\partial X_{k}}=\frac{1}{c\left(\alpha_{k}, x_{k}\right)} \frac{\partial}{\partial x_{k}}
$$

where $c\left(\alpha_{k}, x_{k}\right)$ corresponds to the non-integer dimensionality along the $X_{k}$-axis and it is defined by [39]

$$
c\left(\alpha_{k}, x_{k}\right)=\frac{\pi^{\alpha_{k} / 2}}{\Gamma\left(\alpha_{k} / 2\right)}\left|x_{k}\right|^{\alpha_{k}-1} .
$$

For the case of spatially disordered semiconductor or porous solid, the system can be effectively modeled by replacing the anisotropy with an isotropic continuum in an $\alpha$-dimensional space, with a parameter $0<\alpha \leq 1$ to measure the anisotropy or disorder of the material.

Using the operators in Eq. 27, we can generalize vector differential operators in an $\alpha$-dimensional space. The gradient of a scalar function $\varphi(\mathbf{r})$ in fractional-dimensional space is

$$
\nabla_{\alpha} \varphi(\mathbf{r})=\sum_{k=1}^{3} \mathbf{e}_{k} \partial_{\alpha_{k}, x_{k}} \varphi(\mathbf{r}),
$$

where $\mathbf{e}_{k}$ are unit base vectors of the Cartesian coordinate system. The divergence of the vector field $\mathbf{f}(\mathbf{r})=\mathbf{e}_{k} f_{k}(\mathbf{r})$ is

$$
\nabla_{\alpha} \cdot \mathbf{f}(\mathbf{r})=\sum_{k=1}^{3} \partial_{\alpha_{k}, x_{k}} f(\mathbf{r}) .
$$

The curl for the vector field $\mathbf{f}(\mathbf{r})$ is

$$
\nabla_{\alpha} \times \mathbf{f}(\mathbf{r})=\sum_{k, i, l=1}^{3} \mathbf{e}_{i} \varepsilon_{i k l} \partial_{\alpha_{k}, x_{k}} f(\mathbf{r}),
$$

where $\varepsilon_{i k l}$ is the Levi-Civita symbol. Using Eqs. 29) and (30), the scalar Laplacian in the fractional-dimensional-space is written as [39]

$$
\begin{aligned}
\nabla_{\alpha}^{2} \varphi(\mathbf{r}) & =\nabla_{\alpha} \cdot \nabla_{\alpha} \varphi(\mathbf{r}) \\
& =\sum_{k=1}^{3} \frac{1}{c^{2}\left(\alpha_{k}, x_{k}\right)}\left(\frac{\partial^{2}}{\partial x_{k}^{2}}-\frac{\alpha_{k}-1}{x_{k}} \frac{\partial}{\partial x_{k}}\right) .
\end{aligned}
$$


TABLE II

SUMMARY OF MODIFIED SCLC MODELS FOR SPATIALLY DISORDERED ORGANIC SEMICONDUCTORS PROPOSED IN THIS WORK. SEE MAIN TEXT FOR COMPLETE DESCRIPTION OF TERMINOLOGIES.

\begin{tabular}{lll}
\hline \hline Description & Modified SCLC Model & Eqs. \\
\hline \hline trap-free & $J=\frac{9 \alpha^{3}}{8}\left[\frac{\Gamma(\alpha / 2)}{\pi^{\alpha / 2}}\right]^{3} \epsilon \mu \frac{V_{0}^{2}}{L^{3 \alpha}}$ & $(13)$ \\
$\begin{array}{l}\text { trap-limited } \\
\text { (exponential trap density) }\end{array}$ & $J=N_{c} \mu e^{1-l}\left[\frac{\Gamma(\alpha / 2)}{\pi^{\alpha / 2}}\right]^{2 l+1}\left[\frac{\epsilon \alpha l}{N_{t}(l+1)}\right]^{l} \times\left(\frac{2 \alpha l+\alpha}{l+1}\right)^{l+1} \frac{V_{0}^{l+1}}{L^{2 \alpha l+\alpha}}$ \\
$\begin{array}{l}\text { trap-limited } \\
\text { (Gaussian trap density) }\end{array}$ & $J=N_{c} \mu e^{1-l} \times\left[\frac{\Gamma(\alpha / 2)}{\pi^{\alpha / 2}}\right]^{2 l+1}\left[\frac{\epsilon \alpha l}{N_{t} \exp \left(\left(E_{t c}-E_{a}\right) / k T_{c}\right)(l+1)}\right]^{l} \times\left(\frac{2 \alpha l+\alpha}{l+1}\right)^{l+1} \frac{V_{0}^{l+1}}{L^{2 \alpha l+\alpha}}$ \\
$\begin{array}{l}\text { field-dependent } \\
\text { (exponential) }\end{array}$ \\
$\begin{array}{l}\text { field-dependent } \\
\text { (power-law) }\end{array}$ & $J=\frac{9 \alpha^{3}}{8}\left[\frac{\Gamma(\alpha / 2)}{\pi^{\alpha / 2}}\right]^{3} \epsilon \mu_{0} \frac{V_{0}^{2}}{L^{3 \alpha}} \exp (0.89 \gamma \sqrt{E})$ \\
\hline \hline
\end{tabular}

\section{REFERENCES}

[1] P. Blom, C. Tanase, D. De Leeuw, and R. Coehoorn, "Thickness scaling of the space-charge-limited current in poly (p-phenylene vinylene)," Applied physics letters, vol. 86, no. 9, p. 092105, 2005.

[2] M. Kuik, G.-J. A. Wetzelaer, H. T. Nicolai, N. I. Craciun, D. M. De Leeuw, and P. W. Blom, "25th anniversary article: Charge transport and recombination in polymer light-emitting diodes," Advanced Materials, vol. 26, no. 4, pp. 512-531, 2014.

[3] A. J. Campbell, R. Rawcliffe, A. Guite, J. C. D. Faria, A. Mukherjee, M. A. McLachlan, M. Shkunov, and D. D. Bradley, "Chargecarrier density independent mobility in amorphous fluorene-triarylamine copolymers," Advanced Functional Materials, vol. 26, no. 21, pp. 37203729, 2016.

[4] M. A. Lampert and P. Mark, "Current injection in solids," 1970.

[5] N. F. Mott and R. W. Gurney, Electronic processes in ionic crystals. Clarendon Press, 1948.

[6] P. Mark and W. Helfrich, "Space-charge-limited currents in organic crystals," Journal of Applied Physics, vol. 33, no. 1, pp. 205-215, 1962.

[7] P. Murgatroyd, "Theory of space-charge-limited current enhanced by frenkel effect," Journal of Physics D: Applied Physics, vol. 3, no. 2, p. $151,1970$.

[8] H. Bässler, "Charge transport in disordered organic photoconductors a monte carlo simulation study," physica status solidi (b), vol. 175 , no. 1 , pp. 15-56, 1993.

[9] J. C. Blakesley, F. A. Castro, W. Kylberg, G. F. Dibb, C. Arantes, R. Valaski, M. Cremona, J. S. Kim, and J.-S. Kim, "Towards reliable charge-mobility benchmark measurements for organic semiconductors," Organic Electronics, vol. 15, no. 6, pp. 1263-1272, 2014.

[10] C. Tanase, E. Meijer, P. Blom, and D. De Leeuw, "Unification of the hole transport in polymeric field-effect transistors and light-emitting diodes," Physical Review Letters, vol. 91, no. 21, p. 216601, 2003.

[11] I. Fishchuk, V. Arkhipov, A. Kadashchuk, P. Heremans, and H. Bässler, "Analytic model of hopping mobility at large charge carrier concentrations in disordered organic semiconductors: Polarons versus bare charge carriers," Physical Review B, vol. 76, no. 4, p. 045210, 2007.

[12] W. Pasveer, J. Cottaar, C. Tanase, R. Coehoorn, P. Bobbert, P. Blom, D. De Leeuw, and M. Michels, "Unified description of charge-carrier mobilities in disordered semiconducting polymers," Physical review letters, vol. 94, no. 20, p. 206601, 2005.

[13] J. Cottaar, L. Koster, R. Coehoorn, and P. Bobbert, "Scaling theory for percolative charge transport in disordered molecular semiconductors," Physical review letters, vol. 107, no. 13, p. 136601, 2011.

[14] C. Tanase, P. Blom, D. De Leeuw, and E. Meijer, "Charge carrier density dependence of the hole mobility in poly (p-phenylene vinylene)," physica status solidi (a), vol. 201, no. 6, pp. 1236-1245, 2004.

[15] I. Fishchuk, A. Kadashchuk, J. Genoe, M. Ullah, H. Sitter, T. B. Singh, N. Sariciftci, and H. Bässler, "Temperature dependence of the charge carrier mobility in disordered organic semiconductors at large carrier concentrations," Physical Review B, vol. 81, no. 4, p. 045202, 2010.

[16] I. Katsouras, A. Najafi, K. Asadi, A. Kronemeijer, A. Oostra, L. Koster, D. M. de Leeuw, and P. W. Blom, "Charge transport in poly (p- phenylene vinylene) at low temperature and high electric field," Organic Electronics, vol. 14, no. 6, pp. 1591-1596, 2013.

[17] T. Leijtens, J. Lim, J. Teuscher, T. Park, and H. J. Snaith, "Charge density dependent mobility of organic hole-transporters and mesoporous tio2 determined by transient mobility spectroscopy: Implications to dyesensitized and organic solar cells," Advanced Materials, vol. 25, no. 23, pp. 3227-3233, 2013.

[18] A. Nenashev, J. Oelerich, and S. Baranovskii, "Theoretical tools for the description of charge transport in disordered organic semiconductors," Journal of Physics: Condensed Matter, vol. 27, no. 9, p. 093201, 2015.

[19] N. Tessler, Y. Preezant, N. Rappaport, and Y. Roichman, "Charge transport in disordered organic materials and its relevance to thin-film devices: A tutorial review," Advanced Materials, vol. 21, no. 27, pp. 2741-2761, 2009.

[20] R. Noriega, J. Rivnay, K. Vandewal, F. P. Koch, N. Stingelin, P. Smith, M. F. Toney, and A. Salleo, "A general relationship between disorder, aggregation and charge transport in conjugated polymers," Nature materials, vol. 12, no. 11, pp. 1038-1044, 2013.

[21] M. Zubair, Y. S. Ang, and L. K. Ang, "Fractional fowler-nordheim law for field emission from rough surface with nonparabolic energy dispersion," IEEE Transactions on Electron Devices, vol. 65, no. 6, pp. 2089-2095, 2018.

[22] M. Zubair and L. K. Ang, "Fractional-dimensional child-langmuir law for a rough cathode," Physics of Plasmas (1994-present), vol. 23, no. 7, p. 072118, 2016.

[23] F. H. Stillinger, "Axiomatic basis for spaces with noninteger dimension," Journal of Mathematical Physics, vol. 18, no. 6, pp. 1224-1234, 1977.

[24] C. Palmer and P. N. Stavrinou, "Equations of motion in a non-integerdimensional space," Journal of Physics A: Mathematical and General, vol. 37, no. 27, p. 6987, 2004.

[25] M. Sadallah and S. I. Muslih, "Solution of the equations of motion for einsteins field in fractional d dimensional space-time," International Journal of Theoretical Physics, vol. 48, no. 12, pp. 3312-3318, 2009.

[26] V. E. Tarasov, "Heat transfer in fractal materials," International Journal of Heat and Mass Transfer, vol. 93, pp. 427-430, 2016.

[27] M. Ostoja-Starzewski, J. Li, H. Joumaa, and P. N. Demmie, "From fractal media to continuum mechanics," ZAMM-Journal of Applied Mathematics and Mechanics/Zeitschrift für Angewandte Mathematik und Mechanik, vol. 94, no. 5, pp. 373-401, 2014.

[28] A. S. Balankin and B. E. Elizarraraz, "Map of fluid flow in fractal porous medium into fractal continuum flow," Physical Review E, vol. 85, no. 5, p. 056314, 2012.

[29] M. Zubair, M. J. Mughal, and Q. A. Naqvi, Electromagnetic fields and waves in fractional dimensional space. Springer Science \& Business Media, 2012.

[30] M. J. Mughal and M. Zubair, "Fractional space solutions of antenna radiation problems: An application to hertzian dipole," in Signal Processing and Communications Applications (SIU), 2011 IEEE 19th Conference on. IEEE, 2011, pp. 62-65.

[31] Q. A. Naqvi and M. Zubair, "On cylindrical model of electrostatic potential in fractional dimensional space," Optik-International Journal for Light and Electron Optics, vol. 127, no. 6, pp. 3243-3247, 2016. 
[32] M. Zubair, M. J. Mughal, and Q. A. Naqvi, "An exact solution of the cylindrical wave equation for electromagnetic field in fractional dimensional space," Progress In Electromagnetics Research, vol. 114, pp. 443-455, 2011.

[33] H. Asad, M. J. Mughal, M. Zubair, and Q. A. Naqvi, "Electromagnetic greens function for fractional space," Journal of Electromagnetic Waves and Applications, vol. 26, no. 14-15, pp. 1903-1910, 2012.

[34] H. Asad, M. Zubair, and M. J. Mughal, "Reflection and transmission at dielectric-fractal interface," Progress In Electromagnetics Research, vol. 125, pp. 543-558, 2012.

[35] M. Zubair, M. J. Mughal, and Q. A. Naqvi, "An exact solution of the spherical wave equation in d-dimensional fractional space," Journal of Electromagnetic Waves and Applications, vol. 25, no. 10, pp. 1481-1491, 2011.

[36] M. Zubair, M. J. Mughal, Q. A. Naqvi, and A. A. Rizvi, "Differential electromagnetic equations in fractional space," Progress In Electromagnetics Research, vol. 114, pp. 255-269, 2011.

[37] M. Zubair, M. J. Mughal, and Q. A. Naqvi, "On electromagnetic wave propagation in fractional space," Nonlinear Analysis: Real World Applications, vol. 12, no. 5, pp. 2844-2850, 2011.

[38] M. Zubair, M. J. Mughal, and Q. A. Naqvi, "The wave equation and general plane wave solutions in fractional space," Progress In Electromagnetics Research Letters, vol. 19, pp. 137-146, 2010.

[39] V. E. Tarasov, "Anisotropic fractal media by vector calculus in noninteger dimensional space," Journal of Mathematical Physics, vol. 55, no. 8, p. $083510,2014$.

[40] W. Hwang and K. Kao, "Studies of the theory of single and double injections in solids with a gaussian trap distribution," Solid-State Electronics, vol. 19, no. 12, pp. 1045-1047, 1976.

[41] D. Abbaszadeh, A. Kunz, G. Wetzelaer, J. Michels, N. Cra?ciun, K. Koynov, I. Lieberwirth, and P. Blom, "Elimination of charge carrier trapping in diluted semiconductors," Nature materials, vol. 15, no. 6, pp. 628-633, 2016.

[42] M. Mandoc, B. de Boer, G. Paasch, and P. Blom, "Trap-limited electron transport in disordered semiconducting polymers," Physical Review B, vol. 75, no. 19, p. 193202, 2007.

[43] I. Chen, "A model of charge injection at metal-insulator contacts," Solid State Communications, vol. 26, no. 6, pp. 359-363, 1978.

[44] M. Abkowitz, J. Facci, and M. Stolka, "Time-resolved space chargelimited injection in a trap-free glassy polymer," Chemical physics, vol. 177, no. 3, pp. 783-792, 1993.

[45] H. Nicolai, G. Wetzelaer, M. Kuik, A. Kronemeijer, B. De Boer, and P. Blom, "Space-charge-limited hole current in poly (9, 9dioctylfluorene) diodes," Applied Physics Letters, vol. 96, no. 17, p. 172107, 2010.

[46] G. Horowitz, D. Fichou, X. Peng, and P. Delannoy, "Evidence for a linear low-voltage space-charge-limited current in organic thin films. film thickness and temperature dependence in alpha-conjugated sexithienyl," Journal de Physique, vol. 51, no. 13, pp. 1489-1499, 1990.

[47] R. Coehoorn, S. Vulto, S. Van Mensfoort, J. Billen, M. Bartyzel, H. Greiner, and R. Assent, "Measurement and modeling of carrier transport and exciton formation in blue polymer light emitting diodes," in Photonics Europe. International Society for Optics and Photonics, 2006, pp. $619200-619200$.

[48] M. Mandoc, B. De Boer, and P. Blom, "Electron-only diodes of poly (dialkoxy-p-phenylene vinylene) using hole-blocking bottom electrodes," Physical Review B, vol. 73, no. 15, p. 155205, 2006.

[49] T.-Y. Chu and O.-K. Song, "Hole mobility of n, n'-bis (naphthalen-1-yl)n, n'-bis (phenyl) benzidine investigated by using space-charge-limited currents," Applied physics letters, vol. 90, no. 20, pp. 203 512-203 512, 2007.

[50] K. H. Cheon, J. Cho, B. T. Lim, H.-J. Yun, S.-K. Kwon, Y.-H. Kim, and D. S. Chung, "Analysis of charge transport in high-mobility diketopyrrolopyrole polymers by space charge limited current and time of flight methods," RSC Advances, vol. 4, no. 67, pp. 35344-35347, 2014.

[51] W. Brütting, S. Berleb, and A. Mückl, "Space-charge limited conduction with a field and temperature dependent mobility in alq light-emitting devices," Synthetic Metals, vol. 122, no. 1, pp. 99-104, 2001.

[52] J. John, S. Sivaraman, S. Jayalekshmy, and M. Anantharaman, "Investigations on the mechanism of carrier transport in plasma polymerized pyrrole thin films," Journal of Physics and Chemistry of Solids, vol. 71, no. 7, pp. 935-939, 2010.

[53] A. Boni, I. Pintilie, L. Pintilie, D. Preziosi, H. Deniz, and M. Alexe, "Electronic transport in (la, sr) mno3-ferroelectric-(la, sr) mno3 epitaxial structures," Journal of Applied Physics, vol. 113, no. 22, p. 224103, 2013.
[54] G. A. MacDonald, P. A. Veneman, D. Placencia, and N. R. Armstrong, "Electrical property heterogeneity at transparent conductive oxide/organic semiconductor interfaces: mapping contact ohmicity using conducting-tip atomic force microscopy," ACS nano, vol. 6, no. 11, pp. 9623-9636, 2012.

[55] O. G. Reid, K. Munechika, and D. S. Ginger, "Space charge limited current measurements on conjugated polymer films using conductive atomic force microscopy," Nano letters, vol. 8, no. 6, pp. 1602-1609, 2008.

[56] B. J. West, Fractional calculus view of complexity: Tomorrow's science. CRC Press, 2015

[57] V. E. Tarasov, Fractional dynamics: applications of fractional calculus to dynamics of particles, fields and media. Springer Science \& Business Media, 2011.

[58] K. Falconer, Fractal geometry: mathematical foundations and applications. John Wiley \& Sons, 2004

[59] K. B. Oldham and J. Spanier, The Fractional Calculus. Academic Press, New York, 1974.

[60] G. Calcagni, "Geometry and field theory in multi-fractional spacetime," Journal of High Energy Physics, vol. 2012, no. 1, pp. 1-77, 2012.

[61] A. S. Balankin, "Effective degrees of freedom of a random walk on a fractal," Physical Review E, vol. 92, no. 6, p. 062146, 2015.

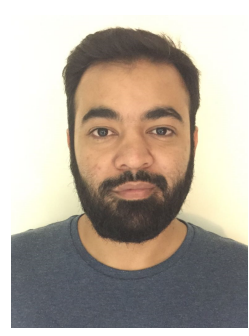

Muhammad Zubair (S'13-M'15) received his $\mathrm{Ph} . \mathrm{D}$. degree in electronic engineering from the Politecnico di Torino, Italy, in 2015. From 2015 to 2017, he was with the SUTD-MIT International Design Center, Singapore. Since 2017, he has been with Information Technology University, Lahore, Pakistan.

His current research interests include charge transport, electron device modeling, computational electromagnetics, fractal electrodynamics, and microwave imaging.

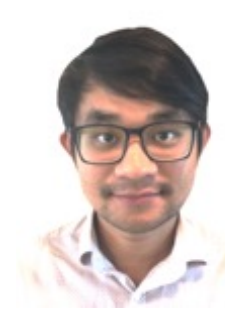

Yee Sin Ang his bachelors degree in medical and radiation physics in 2010, and his $\mathrm{PhD}$ degree in theoretical condensed matter physics in 2014 from the University of Wollongong (UOW), Australia. He is currently a Research Fellow with the Singapore University of Technology and Design, Singapore.

His research interests include the theory and mathematical modelling of electron emission phenomena in $2 \mathrm{D}$ and topological materials, electron transport physics across 2D/3D, 2D material valleytronics, nanoelectronics and superconducting devices.

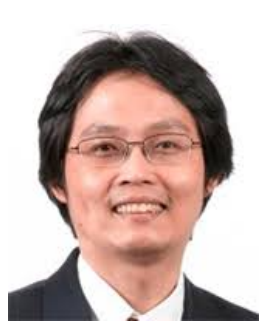

Lay Kee Ang (S'95-M'00-SM'08) received the B.S. degree from the Department of $\mathrm{Nu}$ clear Engineering, National Tsing Hua University, Hsinchu, Taiwan, in 1994, and the M.S. and Ph.D. degrees from the Department of Nuclear Engineering and Radiological Sciences, University of Michigan, Ann Arbor, MI, USA, in 1996 and 1999, respectively. Since 2011, he has been with the Singapore University of Technology and Design, Singapore.

$\mathrm{He}$ is currently the Interim Head and Professor of the Engineering Product Development pillar and also the $\mathrm{Ng}$ Teng Fong Chair Professor of SUTD-ZJU IDEA. 